Literature Reviews

\title{
Object Tracking in Wireless Sensor Networks: Challenges and Solutions
}

\author{
Shereen Ismail, Eman Alkhader and Said Elnaffar \\ ${ }^{1,3}$ Department of Computer Science and Engineering, American University of Ras Al-Khaimah, United Arab Emirates \\ ${ }^{2}$ Department of Electrical Engineering, University of Jordan, Jordan
}

Article history

Received: 03-10-2015

Revised: 27-04-2016

Accepted: 29-04-2016

Corresponding Author:

Shereen Ismail

Department of Computer

Science and Engineering,

American University of Ras Al-

Khaimah, United Arab

Emirates

Mob: +971505373477

Fax: +97172210638

Email: shereen.subhi@aurak.ac.ae

\begin{abstract}
Wireless Sensor Networks (WSNs) are small, inexpensive and battery-operated sensor nodes that are deployed over a geographical area. WSNs are used in many applications such border patrolling, military intrusion detection, wildlife animal monitoring, surveillance of natural disasters and healthcare systems. Mobile object tracking is a vital task in all these applications. The goal of this work is to highlight the most important challenges in the field of object tracking and provide a survey of the WSN architectural design and implementation approaches for tackling this problem. To that end, we analyze how each approach responds to each challenge and where it falls short. This analysis should provide researchers with a state-of-the-art review and inspire them to propose novel solutions.
\end{abstract}

Keywords: Object Tracking Survey, Challenges, Architectures, Approaches Design Issues

\section{Introduction}

A Wireless Sensor Network (WSN) is usually comprised of hundreds of small sensor devices, deployed randomly or manually in order to observe an event of interest. If sensors are in the proximity of the event of interest, they track and report back the observed data to the base station (Sink) periodically. The base station serves as a gateway to remote command centers for further processing and data aggregation (Younis et al., 2014).

The sensor is a small device that consists of four units: Sensing unit; which is responsible for transforming a physical quantity into electrical signal (Sendra et al., 2011), processing unit (or microcontroller and memory unit) that is responsible for functions such as controlling the sensor's activities and executing the communication protocols, communication unit (or wireless radio transceiver) that is used to communicate with the external world and the neighboring nodes and power unit (or the battery) which is usually nonrechargeable and cannot be replaced.

Over the past years, WSNs have proved high effectiveness in numerous applications such as border patrolling, military-restricted areas, prison walls monitoring, campus security and rescue operations, surveillance, wildlife animal tracking, traffic control, home automation and remote healthcare monitoring systems (Tsukamoto et al., 2009; Rault et al., 2014; Prasanna and Rao, 2012).

Border patrol systems, for example, have recently gained lots of interest for the purpose of watching and controlling borders, detecting and tracking intruders, enemy movements or any illegal activities. WSNs also proved their importance in the field of public safety and military applications such as mine field detection and battle field surveillance. Furthermore, WSNs have been used in healthcare systems especially for what is called Body Sensor Network (Sun et al., 2012; Alhmiedat et al., 2012) that is used to monitor the patient's body and gather clinical information to help in rehabilitate physically impaired persons. In traffic-monitoring systems, WSNs are used on streets to collect data about traffic to help people to get the latest information regarding traffic jam in different areas and to achieve intelligent transportation system (Kafi et al., 2013).

The salient task that is common among all these applications is object (or target) tracking. This object could be a fugitive, an intruder at a border, or an attacker at a military base. Tracking these mobile objects involve several sub-tasks such as object interception (or detection), localization and continual reporting of its position to the base station (Tsukamoto et al., 2009; Darabkh et al., 2012). Tracked objects may have different types of signals to be sensed. For example, sensing environmental changes such as light, temperature, pressure and acoustics, or chemical, 
biological and radiological changes in case of security attacks (Chen and Varshney, 2004).

Our objective in this study is to provide new researchers who are interested in object tracking in sensor networks with a reasonably comprehensive review of the field and inspire them to pursue it with new perspectives. To that end, our methodology in this study depends on surveying tens of papers published over the last decade in order to identify the challenges that researchers faced and categorize them. Then, we investigate the various solutions that tackled these challenges and classify them based on (1) the network architecture used and (2) the approach adopted. By this effort, we hope that we serve researchers get the essential background that inspires them to come up with novel solutions.

Our paper is structured as follows. Section 1 defines the common challenges encountered in object tracking research. Section 2 discusses the frequently found network architecture and the most prominent approaches used for object tracking. In section 3, we discuss and analyze our findings. We conclude our paper by section 4 .

\section{Challenges in Object Tracking}

Throughout our research journey (Ismail et al., 2015; Darabkh et al., 2012), we were able to identify several challenges pertaining to object tracking in wireless sensor networks. Next, we present these challenges as categories that are independent of their applications.

\section{Scalability}

Scalability is a twofold challenge: The number of sensor nodes in the network and the number of objects need to be tracked simultaneously. It is not uncommon to have a WSN deployment that consists of thousands of sensor nodes. The number may even reach millions in some applications (Li et al., 2008). With such large number of nodes, it is not easy to attend to each one due to several factors: Nodes many not be physically reachable, nodes may fail and other new ones may join the network. In such unpredictable, dynamic environment, scalable coordination and management functions are necessary to having robust WSNs. Consequently, designers of tracking algorithms are typically concerned about optimization problems germane to the size of the network, efficient scheduling of active vs. inactive nodes, energy consumption and communication overhead among sensor nodes.

Furthermore, the number of objects needs to be tracked manifests another facet of scalability challenges. For example, tracking algorithms should be able to uniquely identify each object moving especially when the number of issued packets is increased for the sake of increasing accuracy (Naderan et al., 2012). They also should be optimized and adopt efficient scheduling mechanisms in order to intercept and track multiple objects simultaneously while being energy-conservative.

\section{Stability}

Since sensor nodes are likely to be installed in harsh conditions outdoors or in hostile environments, they are commonly subject to device failures or may change their initial deployment result from environmental influences such as wind or waterfall (Marks, 2010). Therefore, it is crucial for any object tracking system to demonstrate a reasonable degree of fault-tolerance and adopt some recovery mechanism (Tseng et al., 2003).

\section{Node Deployment}

Depending on the application, the WSN deployment can be either deterministic, where nodes are placed manually in a pre-planned manner (Yick et al., 2008) at certain Cartesian coordinates (Sendra et al., 2011), or randomized, where nodes are deployed across certain geographical area in an ad-hoc manner (Yang and Sikdar, 2003). Compared to random networks, deterministic networks are featured by lower complexity and lower cost of network maintenance and management because their nodes deployed placed at specific locations that ensure coverage. On the other hand, random deployment can spawn uncovered areas (Yick et al., 2008). In addition, location identification for each sensor node is a must after deployment and before putting the network in operation. Location can be determined using Global Positioning System (GPS) system or manually by calculations (Garg and Jhamb, 2013) or by finding the relative location given that each node is within the coverage of another node ( $\mathrm{Li}$ et al., 2014). Unfortunately, the random deployment is the only choice when we need to setup WSNs in harsh, unsafe or hostile ambiences. However, in a new type of WSNs, some sensor nodes reposition themselves over time in order to maintain coverage.

This goal can be achieved by one of two methods. The first method depends on self-deployment where sensors autonomously reposition themselves in order to improve coverage. The second method depends on the relocation of redundant nodes in order to cover for the failed nodes (Zhu et al., 2011).

\section{Computation and Communication Costs}

Any WSN consists of small sensors with constrained capabilities of computation and communication. Typically, the cost of local computation is much lower than communication cost (Ren et al., 2008), which makes reducing the communication overhead a priority for any WSN algorithm.

\section{Energy Constraints}

Due to the difficulty of recharging, the lifetime of the battery in each sensor determines how long it can operate (Peynirci et al., 2014). Therefore, energy conservation should be kept in mind in all cases (Li et al., 2008). 
Usually, algorithms tend to minimize energy consumption by: (1) Scheduling when a node should be in active or sleep state (Yang and Sikdar, 2003), or (2) minimizing the communication and computation cost as much as possible. On the other hand, as the author suggested in (Misra et al., 2015), not all sensors that detect the target are in charge with the tracking process. The algorithm uses the sensor's residual energy to check that this sensor is available for the dwelling time that this target will be within range using a prediction formula. Any sensor node that does not meet this criterion is eliminated from the tracking process.

\section{Data Aggregation}

Data aggregation is a common task in WSNs where data spawned from individual sensors are combined and compressed at an intermediate sensor node before relaying them to the final base station, resulting in a minimal number of transmission packets (Jung et al., 2011a). However, the extent of data aggregation depends on the intra-network spatiotemporal correlation of the signal of interest and the nature of the application (Li et al., 2008; Płaczek and Bernas, 2013). It also depends on functions such as suppression, minimum, maximum and average (Sendra et al., 2011) and other statistical techniques that help discover correlations (Naderan et al., 2012). The problem of data aggregation is more apparent when sensor nodes generate duplicate packets. Therefore, it is imperative for any algorithm to reduce travelling packets in order to have a less channel congestion and lower network latency.

\section{Sensor Technology and Localization Techniques}

Currently there are diverse types of sensors and localization techniques with different accuracies but none of them are highly accurate to be used for all possible WSN application scenarios. The best choice of sensor technology for a specific application is highly reliant on the needed distance range, signal propagation cost, precision, bandwidth etc. For instance, infrared, ultrasonic, electromagnetic, optical and Radio Frequency Identification (RFID) systems are the typically used technologies. The most popular localization techniques are range based which use the distance or angles such as Angle-of-arrival, Time-ofarrival, Time-Difference-of-Arrival, and ReceivedSignal-Strength Indicator (Zhang et al., 2010) for indoor environments while GPS is used for outdoor environment since the line-of-sight is required (Muthukrishnan et al., 2005; Gu et al., 2009).

Other issues that are directly related to algorithm design for tracking an object are.

\section{Tracking Accuracy}

Accuracy of tracking algorithms implies low probability of missing the moving object (Peynirci et al.,
2014), low response latency and low sensitivity to external noise. Furthermore, they should be equipped with a recovery mechanism in case the object is lost.

\section{Reporting Frequency}

Reporting frequency poses a tradeoff between accuracy and energy consumption. Tracking algorithms face the challenge of creating a balance between keeping the base station informed about the movement of the mobile object at certain frequency (Garcia et al., 2010) and preserving energy that can be highly consumed at high communication frequency (Li et al., 2008). The sink node can adjust the reporting frequency during the network progress and transmit the new value in a single broadcast message so each node will adjust its frequency accordingly (Mahmood et al., 2014). In non-sink centric approach, each node can increase its frequency in case of retransmission and as part of object recovery mechanism.

\section{Localization Precision}

The precision of determining object's location by the WSN is proportion to the number of sensors used in the localization process. Generally, to determine the location of an object in 2D space, at least three nodes are required and in 3D space, four nodes are required (Garg and Jhamb, 2013). To that end, object tracking algorithms face the challenging tradeoff between high precision and the need to conserve energy by lowering the number of active nodes participating in the localization process.

\section{Sampling Frequency}

One of the WSN parameters that an object-tracking algorithm may need to consider optimizing is the frequency of sampling, that is, how often a sensor attempts to detect the existence of an object per time unit. It is a parameter that can directly affect the precision of localization. Low sampling rate hides the minor changes in object movements, resulting in lower tracking accuracy or even failing to intercept the object entirely especially if it moves at a high speed. On the other hand, increasing the sampling rate improves the tracking accuracy but drains sensor's battery.

\section{Security}

Security is vital in mission-critical applications. In mission-critical WSNs, sensors are deployed in harsh, unsafe or hostile places where they can be easy targets for intruders who may falsify the collected data. Tracking algorithms need to take care of source authentication, data integrity and confidentiality (Oracevic and Ozdemir, 2014a). Violation of one of these 
security properties can lead to unspeakable risks. For instance, object detection algorithm can be deceived by injecting malicious data into the network, garbling the gathered data or sending phony ones. Therefore, object tracking algorithms, especially those used in sensitive application domains, must keep security vulnerabilities in mind prior to deployment.

\section{Solutions for the Challenges}

The literature is rich with approaches that aim to solve object-tracking challenges from different perspectives and for various goals. In this section, we review the network architectures with emphasis on the prominent approaches used and tracking algorithms that operate on top of each approach. Figure 1 depicts our classification of object-tracking architectures, as elucidated in the following subsections.

\section{The Nä̈ve Architecture}

The naïve architecture is the simplest and the most traditional WSN model in which all sensors are always active trying to intercept and monitor objects in their sensing area and reports to one centralized sink node (Tsukamoto et al., 2009; Ramya et al., 2012). With equal responsibility, each sensor independently observes, processes and transmits the monitored data to the sink node (Fayyaz, 2011). Under this centralized approach, the sink node solely undertakes the heavy computation tasks related to tracking and localizing the monitored objects (Sarna and Zaveri, 2010). Moreover, the more sensors the network has, the more messages are relayed onto the sink node, leading to the increase in communication bandwidth consumption. This model is obviously not fault-tolerant due to the single-point-offailure and its limited scalability (Bhatti and $\mathrm{Xu}, 2009$ ). It usually exhibits the worst energy efficiency because of its heavy communication and computation demands. This renders the naive solution a baseline for comparison with other solutions (Feng et al., 2014).
In Tynan et al. (2009), the authors presented object tracking experiments based on centralized architecture to analyze the network performance when sensors use certain state transition model by examining trade-offs between energy, latency, density and accuracy tradeoffs. The localization techniques chosen for the experiments are: Maximum signal strength localization and weighted average localization. In the weighted average localization, each sensor estimates the location of the object; the larger the value, the greater the effect on the overall location estimation is. In the maximum signal strength localization, the maximum signal value sensed at an active sensor is assigned to the location of the object. An example of this central approach is the work in (Feng et al., 2014) where researchers used a grid network structure. They proposed using real time chain grid heads to relay the sensed data to the sink node while keeping other sensors asleep. Sensor nodes can distributedly decide their sleeping time based on the information from their neighbors. This enables distant nodes to sleep while nodes close to the object remain active. Simulation results of this augmented approach outperform the basic naive approach described earlier.

\section{Tree-Based Architecture}

In an attempt to improve performance, some researchers adopted a sub-graph of the entire set of nodes that has a tree structure. The root of this tree is the closest sensor to the object and other sensors get added or removed as the object moves (Tran and Yang, 2006a). In other words, the tree structure follows the object trajectory (Demigha et al., 2013). This structure reduces energy consumption and communication flow by limiting data transmission from the root to the base station through a particular route. However, as the distance between the root node and the object increases, the rate at which the tree needs to be reconfigured also increases. As a result, tree-based structure is not efficient for tracking high speed objects.

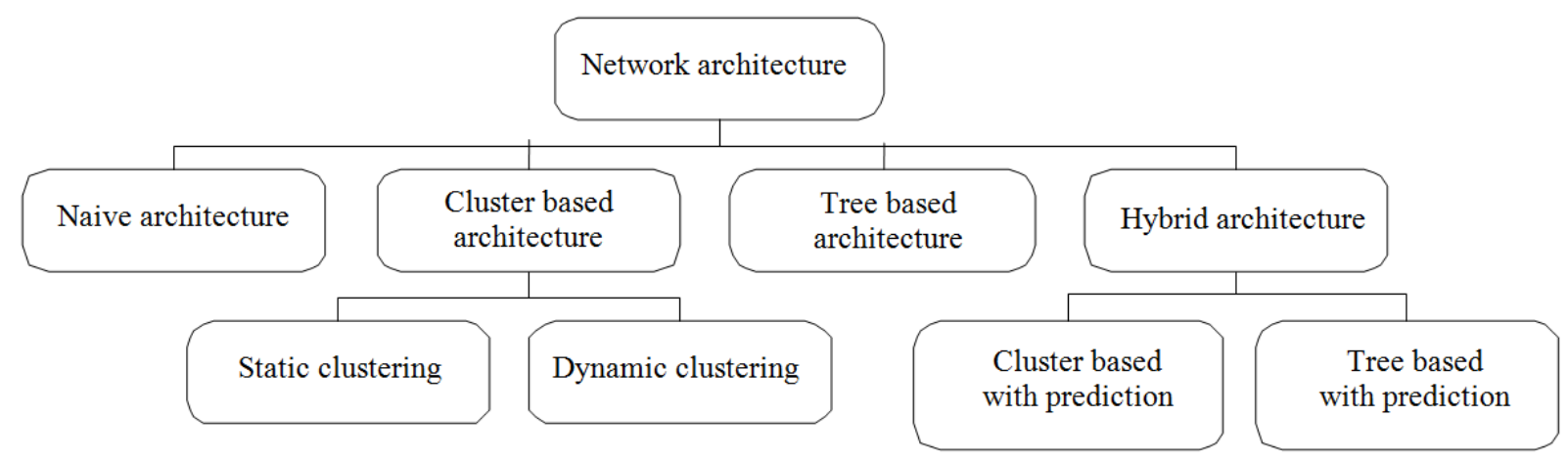

Fig. 1. Network architectures used for object tracking in WSN (Fayyaz, 2011) 
An example of the tree-based approach is the Optimized Communication and Organization (OCO) method that has autonomous characteristics such as self-organizing and auto-routing capabilities throughout the tracking process (Tran and Yang, 2006b). The OCO method consists of four phases: Position finding, processing, tracking and maintenance. A major shortcoming of this method is that sensors must be activated all the time which may lead to the depletion of their batteries.

Shi et al. (2010), the authors proposed an algorithm that constructs a tree of sensors with minimum energy and desirable level of quality at the fusion center. After tree initialization, the algorithm keeps adjusting and reconfiguring the tree in a way that reduces the energy consumption and improves the estimation quality.

\section{Cluster-Based Architecture}

The premise of this architecture (also called two-tier architecture) is to group sensor nodes into clusters in an effort to reduce the number of active nodes. Each cluster has a head sensor and numerous members (Jung et al., $2011 \mathrm{~b}$ ). Instead of reporting to a centralized sink node, cluster members are to report to their cluster head only, which aggregate data and subsequently report to the sink node. To that end, clustering is considered as a hierarchical architecture (Abbasi and Younis, 2007; Gopal and Krishnamoorthy, 2013) that is efficiently used to minimize energy consumption in WSNs when transmitting data from all sensors to the sink node (Heinzelman et al., 2002).

Clustering can be a scalable solution for applications that are comprised of hundreds or thousands of sensor nodes. Scalability requires efficient resource utilization and load balancing in order to increase the network lifetime. Load balancing is achieved by lighter processing load on individual sensor nodes while efficient resource utilization is accomplished through decreasing communication load, reducing possibility of data flow bottlenecks and high survivability as there is no longer a single point of failure. Clustering can be remarkably effective in many-to one, one-to-any, one-tomany, or one-to-all communications (Li et al., 2008). In many-to-one communication, for instance, clustering can reduce communication interference and support data aggregation (Younis and Fahmy, 2004).

In general, any clustering algorithm consists of four main stages:

- Geographical formation of clusters

- Selection of some sensors that are sparsely deployed with high capabilities as cluster heads. The selection is based on their processing capabilities, communication range, residual energy, or location compared to the object. Keep in mind that cluster heads need to be well-distributed over the sensor field to achieve high coverage. Typically, the failure of a cluster head entails re-clustering, however, some approaches can adapt the network topology by resorting to backup cluster heads (Younis et al., 2014)

- Data aggregation stage in which the sensed data are gathered and combined in a less number of packets in preparation to be sent to the cluster heads (Jung et al., 2011a; Sinha and Lobiyal, 2013). Basically, sensor nodes will provide their sensing information upon request (Suganya, 2008)

- Data transmission stage which involves the transfer of the aggregated data from the cluster heads to the sink node

Based on the formation style of clusters, they are classified into static and dynamic, as explained next.

\section{Static Clustering}

In static clustering, clusters are formed statically at the network deployment time as shown in Fig. 2. The attributes of each cluster, such as the cluster size, the coverage area, the sensor members and the cluster head are static (Li and Zhou, 2011). This means that the sensor nodes remain hooked up to the same cluster head throughout the network lifetime (Fayyaz, 2011).

When the object enters a cluster area, the cluster head gets activated and it subsequently activates its cluster members to keep localizing and tracking the detected object. When the object departs the cluster vicinity to another, the current cluster head informs the new one to keep tracking the object (Darabkh et al., 2012).

Despite the simplicity of this cluster architecture, it suffers from several shortcomings. First, it is not faulttolerant due to the fixed membership. If a cluster head goes down, for battery depletion for example, all the sensors in the cluster become useless. Second and due to fixed membership, sensor nodes in different clusters cannot share information and collaborate on data processing (Gopal and Krishnamoorthy, 2013). Finally, fixed membership prevents the adaptability to dynamic scenarios in which nodes in the region of high (low) event concentration may stay active (go to sleep) state (Li and Zhou, 2011).

\section{Dynamic Clustering}

While static clustering is formed at the network designtime, the construction of adaptive clusters is triggered by a special event of interest, such as the acoustic sounds of a moving object, as shown in Fig. 3. When a sensor, hopefully the one that is the nearest to the object or the one with the highest energy, detects an object, it volunteers to play the role of a cluster head (Abbasi and Younis, 2007; Gopal and Krishnamoorthy, 2013). 


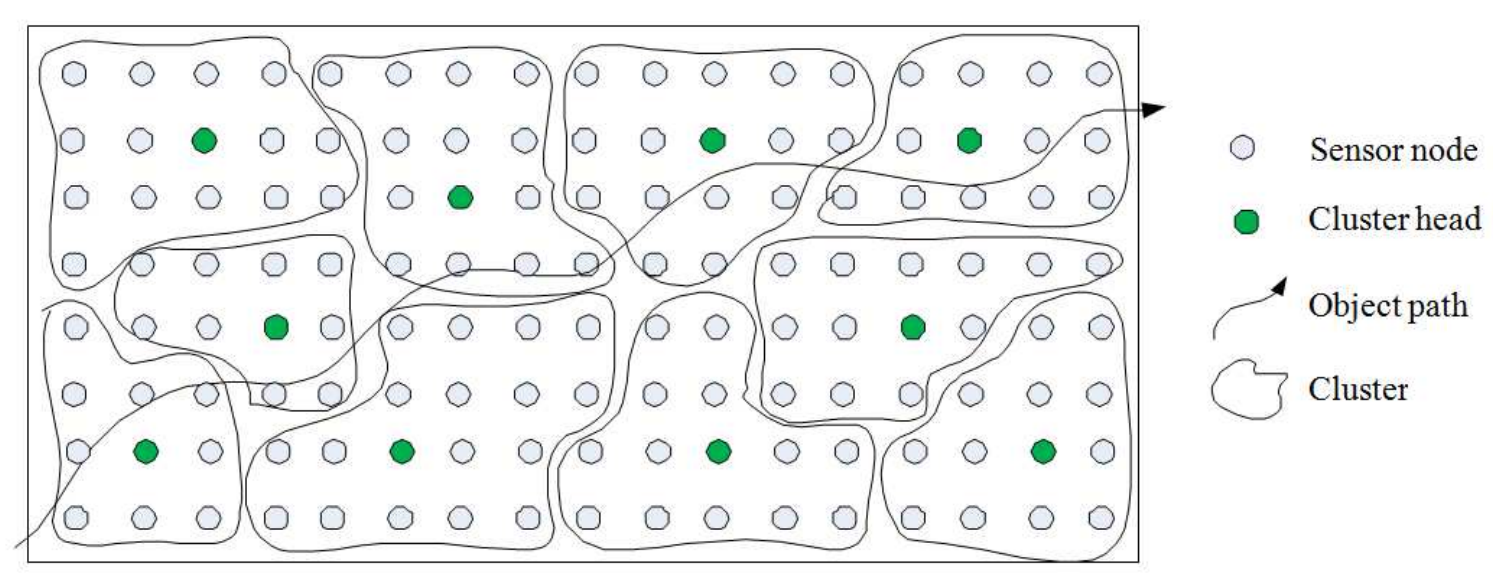

Fig. 2. Static clustering scenario (Ren et al., 2008)

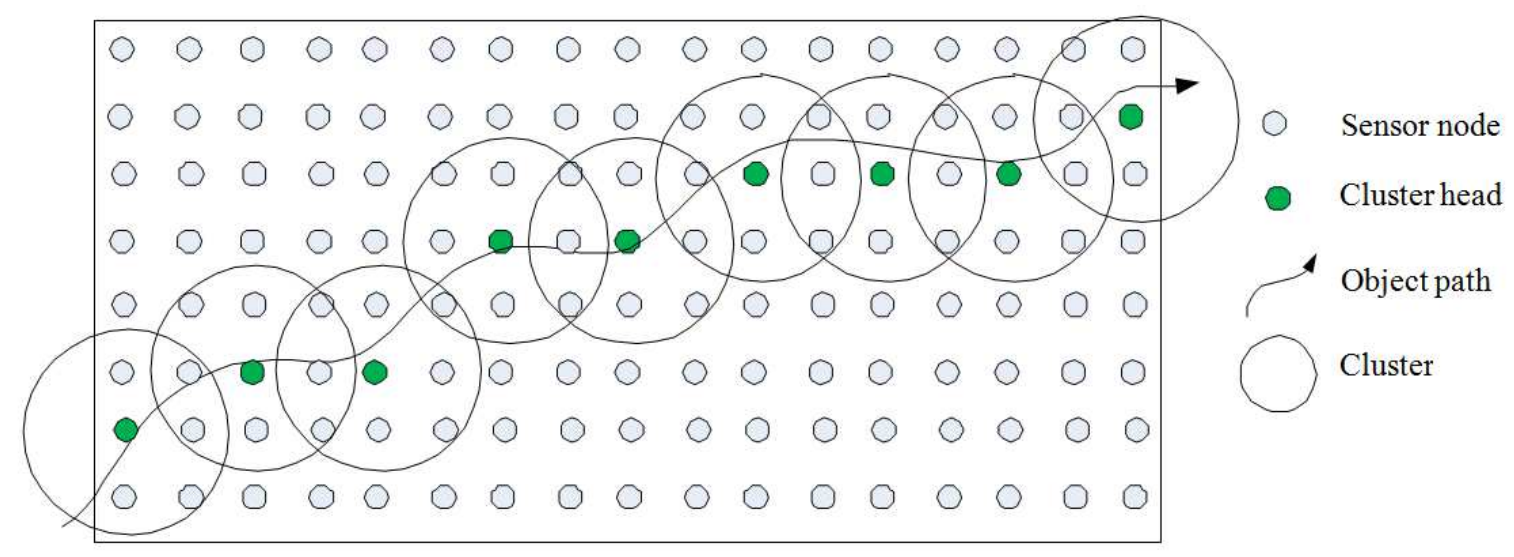

Fig. 3. Dynamic clustering scenario (Jin et al., 2006)

Typically, multiple sensor nodes may detect the event of interest so multiple volunteers may exist. For this reason, some mechanism is used to ensure the selection of only one sensor as a cluster head. Nodes that are close to the cluster head are invited, as members, to form a cluster and report their collected data to the head (Jin et al., 2006). The cluster is dismantled when the object is no longer sensed (Jung et al., 2011a).

Unlike static clustering, nodes in a dynamic cluster may belong to different clusters at different times contingent to object movements. Furthermore, since only one cluster is active within the vicinity of the object with high probability, redundant data are suppressed, leading to better tracking quality. Furthermore, energy consumption is reduced since one cluster is active at a time in accordance to object movement (Gopal and Krishnamoorthy, 2013).

Generally, dynamic clustering is preferred when the WSN is required to cover a large area (Oracevic and Ozdemir, 2014b), while with dense networks, static clustering is more desirable in order to avoid cluster overlapping and the high frequency of cluster head election that occur in the adaptive clustering technique.

In Darman and Ithnin (2014), the authors have mentioned that cluster-based approaches provide better bandwidth utilization and higher scalability than other approaches. They also classify the cluster-based approaches into static, which has a pre-built backbone infrastructure and dynamic that is more suitable with highly dynamic scenarios. In Yan and Wang (2010), the authors have presented a dynamic cluster formation algorithm for object tracking in WSNs. When an object moves towards the sensing field, cluster heads are selected randomly by a certain algorithm which is considered an extension from the Low Energy Adaptive Clustering Hierarchy (LEACH) protocol (Heinzelman et al., 2002). The cluster heads then invite their neighboring sensor nodes to form a cluster. The major advantage of such algorithm is the dynamic number of sensors within each formed cluster. When the object moves around, the formed cluster changes and the set of sensors vary with time. However, the proposed algorithm lacks simulation proof and the random selection of cluster heads most 
likely will not achieve the required tracking accuracy for such a system. An interesting combination of static and dynamic clustering technique was proposed by (Jung et al., 2011a). This mechanism switches to the appropriate clustering technique and aggregation mechanism depending on the network state.

In Darabkh et al. (2012), the authors proposed three cluster-based algorithms for object tracking: Static head, adaptive head and selective static head. Static and selective static head schemes are based on static clustering while adaptive head is based on dynamic clustering. They reached promising tracking accuracy and energy preservation by selecting asking nearby nodes to pitch in the tracking process while leaving the others in a sleep state. They showed that the adaptive head is the most efficient scheme with respect to energy consumption, while static and selective static schemes are better for lowering tracking error especially when the object moves fast.

In Wang et al. (2013), a hybrid cluster-based object tracking approach was proposed that integrate static with on-demand dynamic clustering to manage the tracking task. While static clusters are confined to share information within cluster vicinity, on-demand dynamic clustering, on the other hand, is used when the object enters and exits the boundary region so sensors from different static clusters that intercept the object can temporarily share information. In the same context of solving the boundary problem, the authors in (Akter et al., 2015) proposed to combine static clustering with another incremental clustering algorithm to track an object consistently. In other words, incremental clusters are constructed at the boundaries of static clusters to continue the tracking task. The proposed algorithm performs better in tracking the moving object at the boundary regions than other typical tracking protocols.

\section{Hybrid Architecture}

Hybrid architecture generally combines one of the previously mentioned architectures with some prediction mechanism. Prediction relies on heuristics and attempts to anticipate the upcoming position of the moving object based on its historical positions observed over time and the spatial and temporal knowledge of sensors (Zhenga et al., 2014). Based on this prediction, sensor nodes get scheduled to be either active or asleep (Ren et al., 2008) during each defined time step (Mirsadeghi and Mahani, 2014). Due to the inevitable prediction mistakes, these algorithms have recovery mechanisms in order to make up for the inaccuracy of object localization. Unfortunately, such algorithms are typically too complex to be implemented on sensor nodes with constrained resources.

An example of a hybrid approach is the Hierarchical Prediction Strategy (HPS) that augments the clusterbased approach with a prediction mechanism. In the
HSP strategy, the cluster is built using Voronoi division and the mobile object's next location is predicted (Wang et al., 2008). One of the major shortcomings of such algorithms is the additional complexity resulting from combining the two approaches. Furthermore, the performance overhead incurred was not assessed.

In Raza et al. (2009), the authors presented the Dead Reckoning object tracking protocol that depends on predicting its position by analyzing the time series of historical locations over a time window. Using a position fix technique, the Dead Reckoning protocol provides a mechanism for error avoidance and error correction. The performance of the proposed scheme was assessed in terms of node sleep time, tracking error and object loss.

In Mirsadeghi and Mahani (2014), the author presented a high-precision and energy efficient tracking scheme that is based on clustering architecture and object speed prediction. In each time step, just a few nodes in the vicinity of the predicted position of object get activated as tracker nodes by considering three parameters: distance, remaining energy of nodes and energy needed to send a packet to the cluster head while others remain in power-saving mode. Simulation results showed that the energy consumption of the nonprediction method is too high in comparison with its counterpart prediction one.

An adaptive sensor activation algorithm for target tracking in WSNs is presented in (Zhenga et al., 2014) where the authors used an auction mechanism for selecting the cluster head. In each iteration of the tracking operation, the cluster head tries to predict the region where that target may move to. Based on this predicted region, only nodes within this region are activated and the rest remain asleep. The presented algorithm has proven itself in terms of the network lifetime, energy efficiency and accuracy of tracking.

\section{Tracking Multiple Objects}

Tracking the path of multiple objects is more challenging than tracking a single one due to the need of identifying each object moving in different directions with different speeds and the need of track continuity with good performance. If all energyrestricted nodes are kept active for the purpose of tracking multiple objects, the network traffic and the probability of failure will increase dramatically. Consequently, more complex routing algorithms and energy minimization techniques have to be used. In addition, as each sensor node is responsible for detecting and tracking multiple objects, it should be able to distinguish objects by some means of signal processing algorithms (Naderan et al., 2013). In literature, we can find many object classification algorithms (Panda et al., 2014; Pannetier et al., 2015) that adopt a set of weighted features for the purpose of identifying objects. 


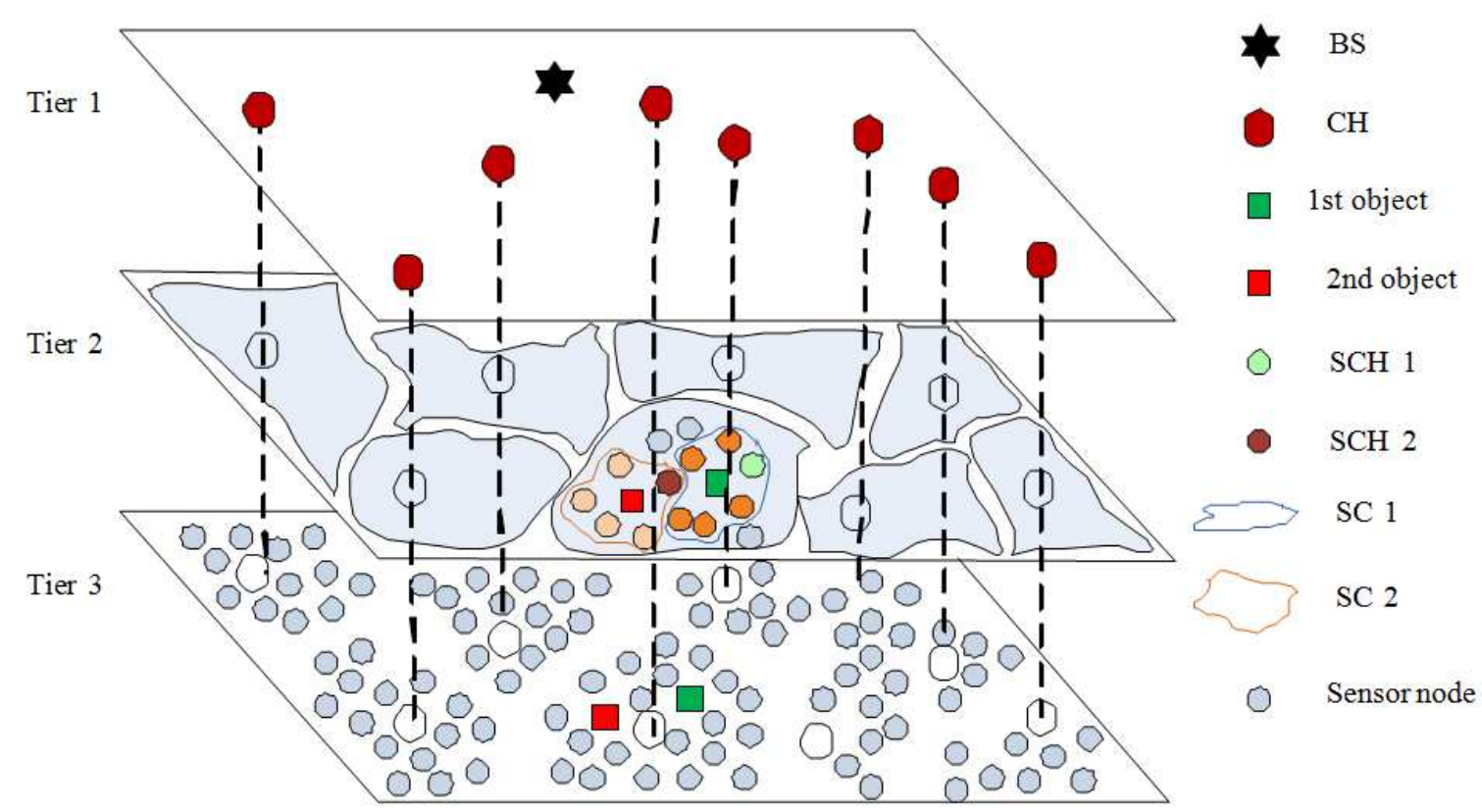

Fig. 4. Three-tier cluster-based WSN

Object's kinematic characteristics such as its movement pattern, position, velocity and acceleration are usually used in tracking multiple objects to narrow the tracking region (Rahman et al., 2010). Dense networks are often used to monitor multiple objects in order to maximize the number of sensors that cover all points in the object's area. In such networks, eliminating redundancy is imperative for efficiency. This can be achieved by using hierarchical multi-tier networks or eventtriggered solutions.

As shown in Fig. 4, three-tier cluster-based network is illustrated where the pre-determined cluster heads keep listening to the medium for any approaching objects then they activate their cluster members based on certain criteria to minimize network traffic and energy consumption. From these members, multiple Sub Cluster Heads (SCHs) could be elected in case of the availability of multiple objects within the same cluster vicinity. The criteria could be a weighted average of multiple factors like the node's remaining energy, the Euclidean distance between the node and the object and the type of sensor or sensor technology (if the network is heterogeneous). If multiple objects are in the vicinity of a certain cluster, we can assign one $\mathrm{SCH}$ for each object. These Sub Clusters (SCs) will detect and localize the objects and send their observation to the upper tier cluster heads up to the end base station.

\section{Summary and Conclusion}

Based on the above survey and analysis and regardless the type of the object to be tracked and the signal to be sensed, we found that most tracking algorithms in WSNs share the following characteristics:

- An efficient algorithm strives to reduce the number of continually active sensor nodes in order to conserve energy

- Any tracking system should report the position of the object to the base station in a timely fashion

- Tracking algorithms care about eliminating (or reducing) correlated, inconsistent, or redundant data in order to reduce not only the packets transferred but also the number of collisions and interference in the shared medium. For the same purpose, sensors should collaborate on processing the data then sending them aggregated to the base station

We also found that the majority of object tracking algorithms aim to tackle the challenge of creating a balance among network resources like communication bandwidth, energy and tracking accuracy. Table 1 presents the various object tracking architectures discussed in this study and compares between them based on how each one tackles the challenges discussed above. Each entry in the table reflects how effective a given approach at tackling the challenge. The level of effectiveness of a given approach with respect to a particular challenge is expressed as either limited, low, moderate, high and applied but with constraints. By studying this table we can see that the cluster-based architecture apparently provides more scalability, stability, energy-efficiency and tracking accuracy than other approaches. 


\begin{tabular}{|c|c|c|c|c|c|c|c|c|c|}
\hline $\begin{array}{l}\text { Approach } \\
\text { /Challenge }\end{array}$ & Scalability & Stability & $\begin{array}{l}\text { Node } \\
\text { deployment }\end{array}$ & $\begin{array}{l}\text { Computation and } \\
\text { communication cost }\end{array}$ & $\begin{array}{l}\text { Energy } \\
\text { constraints }\end{array}$ & $\begin{array}{l}\text { Data } \\
\text { aggregation }\end{array}$ & $\begin{array}{l}\text { Tracking } \\
\text { accuracy }\end{array}$ & $\begin{array}{l}\text { Reporting } \\
\text { frequency }\end{array}$ & $\begin{array}{l}\text { Data } \\
\text { precision }\end{array}$ \\
\hline $\begin{array}{l}\text { Centralized } \\
\text { (Naive) }\end{array}$ & $\mathrm{Li}$ & $\mathrm{Li}$ & $\begin{array}{l}\text { Randomize is } \\
\text { more applicable }\end{array}$ & $\mathrm{H}$ & N/A & N/A & $\mathrm{H}$ & $\begin{array}{l}\mathrm{H} \text { (no real time } \\
\text { constraints) }\end{array}$ & $\mathrm{H}$ \\
\hline Tree based & $\mathrm{M}$ & $\mathrm{M}$ & Randomize & M & M & M & M & $\begin{array}{l}\text { Constraints } \\
\text { can be applied }\end{array}$ & M \\
\hline Cluster based & & $\mathrm{H}$ & $\begin{array}{l}\text { Randomize } \\
\text { or deterministic }\end{array}$ & $\mathrm{L}$ to $\mathrm{M}$ & $\mathrm{L}$ to $\mathrm{M}$ & $\mathrm{H}$ & $\mathrm{M}$ to $\mathrm{H}$ & $\begin{array}{l}\text { Constraints } \\
\text { can be applied }\end{array}$ & $\mathrm{M}$ to $\mathrm{H}$ \\
\hline Hybrid & M & $\mathrm{Li}$ & $\begin{array}{l}\text { Randomize is } \\
\text { more applicable }\end{array}$ & M & M & M & M & $\begin{array}{l}\text { Constraints } \\
\text { can be applied }\end{array}$ & $\mathrm{L}$ to $\mathrm{M}$ \\
\hline
\end{tabular}

The case of multiple objects tracking bears its own set of extra challenges as the locations of multiple objects have to be tracked simultaneously. We have emphasized that increasing the number of objects to be tracked increases the network traffic. Consequently, more complex routing schemes and energy minimization techniques have to be adopted in order to retain an acceptable network performance. Based on these challenges, we suggested future multi object three-tier network based on clustering architecture. Other open issues are:

- How to deploy and manage heterogeneous nodes

- How to deal with node failure and adjust the network topology accordingly

- Study the mobility effects (target, nodes or sink relocation) on the quality of tracking performance and what is required for network adaption accordingly

- The use of efficient aggregation techniques

- The manufacturing of sensor nodes to have more powerful batteries, fast processors and long-distance transceivers in order to optimize the energy consumption and achieve better coverage (Can and Demirbas, 2013).

\section{Funding Information}

Special thanks to the American University of Ras Al Khaimah for providing incentive fund for this research work and for covering the fees of its publication.

\section{Author's Contributions}

Shereen Ismail: Participated in all conception and surveying the literature, data acquisition and analysis and coordinating the work among co-authors.

Eman Alkhader: Contributed to the data gathering task and collaborated with the first author in designing the art work and analyzing the data.

Dr. Said EInaffar: Prominently responsible for the analytical part of the work. He also contributed to summarizing the findings spawn from contrasting approaches with challenges. Dr. Elnaffar heavily participated in the writing and proofreading of this manuscript.

\section{Ethics}

This article is original and contains unpublished material. The corresponding author confirms that all of the other authors have read and approved the manuscript and no ethical issues involved.

\section{References}

Abbasi, A. and M. Younis, 2007. A survey on clustering algorithms for wireless sensor networks. J. Comput. Commun., 3: 2826-2841.

DOI: 10.1016/j.comcom.2007.05.024

Akter, M., M.O. Rahman, M.N. Islam and M.A. Habib, 2015. Incremental clustering-based object tracking in wireless sensor networks. Proceedings of the International Conference on Networking Systems and Security, Jan. 5-7, IEEE Xplore Press, Dhaka, pp: 1-6.

DOI: $10.1109 /$ NSysS.2015.7043534

Alhmiedat, T., A.A. Taleb and M. Bsoul, 2012. A study on threats detection and tracking systems for military applications using WSNs. Int. J. Comput. Applic., 40: 12-18. DOI: 10.5120/5055-7347

Bhatti, S. and J. Xu, 2009. Survey of target tracking protocols using wireless sensor network. Proceedings of the 5th IEEE Conference on Wireless and Mobile Communications, Aug. 23-29, IEEE Xplore Press, Cannes, La Bocca, pp: 110-115. DOI: 10.1109/ICWMC.2009.25

Can, Z. and M. Demirbas, 2013. A survey on in-network querying and tracking services for wireless sensor networks. Ad Hoc Netw., 11: 596-610. DOI: 10.1016/j.adhoc.2012.08.007

Chen, D. and P. Varshney, 2004. QoS support in wireless sensor networks: A survey. Proceedings of the Citeseer International Conference on Wireless Networks, Jun. 21-24, Las Vegas, Nevada, USA, pp: 1-7.

Darabkh, K.A., S.S. Ismail, M. Al-Shurman, I.F. Jafar and E. Alkhader et al., 2012. Performance evaluation of selective and adaptive heads clustering algorithms over wireless sensor networks. J. Netw. Comput. Applic., 35: 2068-2080.

DOI: $10.1016 /$ j.jnca.2012.08.008 
Darman, R. and N. Ithnin, 2014. Object tracking methods in wireless sensor network: Network structure classification. Proceedings of the International Conference on IT Convergence and Security, Oct. 28-30, IEEE Xplore Press, Beijing, pp: 1-3. DOI: 10.1109/ICITCS.2014.7021793

Demigha, O., W. Hidouci and T. Ahmed, 2013. On energy efficiency in collaborative target tracking in wireless sensor network: A review. IEEE Commun. Surveys Tutorials, 15: 1210-1222. DOI: $10.1109 /$ SURV.2012.042512.00030

Fayyaz, M., 2011. Classification of object tracking techniques in wireless sensor networks. J. Wireless Sensor Netw., 3: 121-124.

DOI: $10.4236 /$ wsn.2011.34014

Feng, J., B. Lian and H. Zhao, 2014. Smart power management and delay reduction for target tracking in wireless sensor networks. J. Electr. Comput. Eng. DOI: $10.1155 / 2014 / 641720$

Garcia, O., A. Quintero and S. Pierre, 2010. A global profile-based algorithm for energy minimization in object tracking sensor networks. Elsevier J. Comput. Commun., 33: 736-744.

DOI: 10.1016/j.comcom.2009.11.020

Garg, V. and M. Jhamb, 2013. A review of wireless sensor network on localization techniques. Int. J. Eng. Trends Technol., 4: 1049-1053.

Gopal, K. and R. Krishnamoorthy, 2013. Analysis of cluster based target tracking in wireless sensor networks. Int. J. Comput. Applic., 62: 10-13. DOI: 10.1016/j.comcom.2007.05.024

$\mathrm{Gu}$, Y., A. Lo and I. Niemegeers, 2009. A survey of indoor positioning systems for wireless personal networks. IEEE Commun. Surveys Tutorials, 11: 13-32. DOI: 10.1109/SURV.2009.090103

Heinzelman, W.R., A. Chandrakasan and H. Balakrishnan, 2002. An application-specific protocol architecture for wireless microsensor networks. IEEE Trans. Wireless Commun., 1: 660-670. DOI: 10.1109/TWC.2002.804190

Ismail, S.S., E.I. Al Khader and K.A. Darabkh, 2015. Static clustering for target tracking in wireless sensor networks. Global J. Technol., 8: 167-173.

Jin, G., X. Lu and M. Park, 2006. Dynamic clustering for object tracking in wireless sensor networks. Proceedings of the 3rd International Symposium on Ubiquitous Computing Systems, Oct. 11-13, Seoul, Korea, pp: 200-209. DOI: 10.1007/11890348_16

Jung, W.S., K.W. Lim, Y. Ko and S. Park, 2011 a. Efficient clustering-based data aggregation techniques for wireless sensor networks. Springer J. Wireless Netw., 17: 1387-1400.

DOI: $10.1007 / \mathrm{s} 11276-011-0355-6$

Jung, W.S., K.W. Lim, Y.B. Ko and S. Park, 2011 b. Efficient clustering-based data aggregation techniques for wireless sensor networks. J. Wireless Netw., 17: 1387-1400.

DOI: $10.1007 / \mathrm{s} 11276-011-0355-6$
Kafi, M.A, Y. Challalc, D Djenouria, M. Doudoua, A. Bouabdallahc and N. Badachea, 2013. A study of wireless sensor networks for urban traffic monitoring: Applications and architectures. Proc. Comput. Sci., 19: 617-626.

DOI: $10.1016 /$ j.procs.2013.06.082

Li, J. and Y. Zhou, 2011. Target tracking in wireless sensor networks: Application-centric design.

Li, S., Z. Qin, L. Shan, R. Zhang and X. Yang, 2014. A survey on target tracking in well-deployed wireless sensor networks. J. Software, 9: 1255-1262. DOI: $10.4304 /$ jsw.9.5.1255-1262

Li, Y., T. Thai and W. Wu, 2008. Wireless Sensor Networks and Applications. 1st Edn., Springer Science and Business Media, New York, ISBN-10: 0387495924, pp: 464.

Mahmood, M.A., W.K.G. Seah and I. Welch, 2014. Reliability in wireless sensor networks: A survey and challenges ahead. Comput. Netw., 79: 166-187. DOI: $10.1016 /$ j.comnet.2014.12.016

Marks, M., 2010. A survey of multi-objective deployment in wireless sensor networks. J. Telecommun. Inform. Technol.

Mirsadeghi, M. and A. Mahani, 2014. Energy efficient fast predictor for WSN-based target tracking. Annals Telecommun., 70: 63-71. DOI: $10.1007 / \mathrm{s} 12243-014-0430-y$

Misra, S., A. Singh, S. Chatterjee and A.K. Mandal, 2015. QoS-aware sensor allocation for target tracking in sensor-cloud. Ad Hoc Netw., 33: 140-153. DOI: $10.1016 /$ j.adhoc.2015.04.009

Muthukrishnan, K., M.E. Lijding and P.J.M. Havinga, 2005. Towards smart surroundings: Enabling techniques and technologies for localization. Proceedings of the International Workshop on Location-and Context-Awareness, May 12-13, Oberpfaffenhofen, Germany, pp: 350-362.

DOI: $10.1007 / 1142664632$

Naderan, M., M. Dehghan and H. Pedram, 2013. Upper and lower bounds for dynamic cluster assignment for multi-target tracking in heterogeneous WSNs. J. Parallel Distrib. Comput., 73: 1389-1399. DOI: $10.1016 /$ j.jpdc.2013.04.007

Naderan, M., M. Dehghan, H. Pedram and V. Hakami, 2012. Survey of mobile object tracking protocols in wireless sensor networks: A network-centric perspective. Int. J. Ad Hoc Ubiquitous Comput., 11: 34-63. DOI: 10.1504/IJAHUC.2012.049283

Oracevic, A. and S. Ozdemir, 2014a. A survey of secure target tracking algorithms for wireless sensor networks. Proceedings of the World Congress on Computer Applications and Information Systems, Jan. 17-19, IEEE Xplore Press, Hammamet, pp: 1-6. DOI: 10.1109/WCCAIS.2014.6916628 
Oracevic, A. and S. Ozdemir, 2014b. A survey of secure target tracking algorithms for wireless sensor networks. Proceedings of the World Congress on Computer Applications and Information Systems, Jan. 17-19, IEEE Xplore Press, Hammamet, pp: 1-6. DOI: 10.1109/WCCAIS.2014.6916628

Panda, S., P.S. Chatterjee, I. Das and M. Roy, 2014. Energy efficient multiple objects sensing and classification system in WSNs. Int. J. Recent Trends Eng. Technol., 11: 633-641.

Pannetier, B., J. Moras, J. Dezert, L. Canevet and D. Cosson, 2015. Ground target tracking and classification in an unattended wireless sensor network.

Peynirci, G., I. Korkmaz and M. Gurgen, 2014. An energy conservative wireless sensor network model for object tracking. Proceedings of the Federated Conference on Computer Science and Information Systems, Sept. 7-10, IEEE Xplore Press, Warsaw, pp: 1049-1057. DOI: 10.15439/2014F221

Płaczek, B. and M. Bernas, 2013. Uncertainty-based information extraction in wireless sensor networks for control applications. Ad Hoc Netw., 14: 106-117. DOI: 10.1016/j.adhoc.2013.11.009

Prasanna, S. and S. Rao, 2012. An overview of wireless sensor networks applications and security. Int. J. Soft Comput. Eng., 2: 538-540.

Rahman, A.A.U., M. Naznin and M.A.I. Mollah, 2010. Energy-efficient multiple targets tracking using target kinematics in wireless sensor networks. Proceedings of the 4th International Conference on Sensor Technologies and Applications, Jul. 18-25, IEEE Xplore Press, Venice, pp: 275-280. DOI: $10.1109 /$ SENSORCOMM.2010.101

Ramya, K., K.P. Kumar and V.S. Rao, 2012. A survey on target tracking techniques in wireless sensor networks. Int. J. Comput. Sci. Eng. Survey, 3: 93-108. DOI: 10.5121/ijcses.2012.3408

Rault, T., A. Bouabdallah and Y. Challal, 2014. Energy efficiency in wireless sensor networks: A top-down survey. Elsevier, 67: 104-122.

DOI: 10.1016/j.comnet.2014.03.027

Raza, M.T., G. Bag, S. Yoo and H. Kim, 2009. Dead reckoning based target tracking in wireless sensor networks. Proceedings of the 2nd ACM International Workshop on Adaptive and Reconfigurable Embedded Systems, (RES' 09), ACM New York, NY, USA. DOI: 10.1145/1851340.1851342

Ren, Q., H. Gao, S. Jiang and J. Li, 2008. An energyefficient object tracking algorithm in sensor networks. Springer J. Wireless Algorithms, Syst. Applic., 5258: 237-248.

DOI: $10.1007 / 978-3-540-88582-5 \_24$

Sarna, S. and M. Zaveri, 2010. ERTĀ: Energy efficient real time target tracking approach for wireless sensor networks. Proceedings of the 4th IEEE International Conference on Sensor Technologies and Applications, Jul. 18-25, IEEE Xplore Press, Venice, pp: 220-225. DOI: $10.1109 /$ SENSORCOMM.2010.41
Sendra, S., J. Lloret, M. García and J. Toledo, 2011. Power saving and energy optimization techniques for wireless sensor neworks. Acad. Pub. J. Commun., 6: 439-459. DOI: $10.4304 /$ jcm.6.6.439-459

Shi, L., A. Capponi, K.H. Johansson and R.M. Murray, 2010. Resource optimisation in a wireless sensor network with guaranteed estimator performance. IET Control Theory Applic., 4: 710-723. DOI: 10.1049/iet-cta.2009.0104

Sinha, A. and D.K. Lobiyal, 2013. Performance evaluation of data aggregation for cluster-based wireless sensor network. Human-Centric Comput. Inform. Sci.

Suganya, S., 2008. A cluster-based approach for collaborative target tracking in wireless sensor networks. Proceedings of the 1st IEEE International Conference on Emerging Trends in Engineering and Technology, Jul. 16-18, IEEE Xplore Press, Nagpur, Maharashtra, pp: 276-281.

DOI: 10.1109/ICETET.2008.241

Sun, Z., P. Wanga, M.C. Vuran, M.A. Al-Rodhaan and A.M. Al-Dhelaan et al., 2012. BorderSense: Border patrol through advanced wireless sensor networks. Ad Hoc Networks, 9: 468-477.

Tran, S.P. and T.A. Yang, 2006a. Evaluations of target tracking in wireless sensor networks. Proceedings of the ACM 37th SIGCSE Technical Symposium on Computer Science Education, Mar. 01-05, Houston, TX, USA, pp: 97-101. DOI: $10.1145 / 1121341.1121374$

Tran, S.P.M. and T.A. Yang, 2006b. OCO: Optimized communication \&amp; organization for target tracking in wireless sensor networks. Proceedings of the IEEE International Conference on Sensor Networks, Ubiquitous and Trustworthy Computing, Jun. 5-7, IEEE Xplore Press, Taichung, pp: 428-435. DOI: 10.1109/SUTC.2006.1636209

Tseng, Y., S. Kuo, H. Lee and C. Huang, 2003. Location tracking in a wireless sensor network by mobile agents and its data fusion strategies. Proceedings of ACM International Conference on Information Processing in Sensor Networks, Apr. 22-23, Palo Alto, CA, USA, pp: 625-641. DOI: $10.1007 / 3-540-36978-3 \_42$

Tsukamoto, K., H. Ueda, H. Tamura, K. Kawahara and Y. Oie, 2009. Design of wireless sensor network for multi-point surveillance of a moving target based on the relationship between tracking probability and sensor density. Proceedings of the IEEE 6th Consumer Communications and Networking Conference, Jan. 10-13, IEEE Xplore Press, Las Las Vegas, NV, pp: 1-5.

DOI: $10.1109 / \mathrm{CCNC} .2009 .4784913$ 
Tynan, R., G.M.P. O'Hare, M.J. O'Grady and C. Muldoon, 2009. EDLA tradeoffs for wireless sensor network target tracking. Proceedings of the 29th IEEE International Conference on Distributed Computing Systems, Jun. 22-26, IEEE Xplore Press, Montreal, QC, pp: 440-446.

DOI: $10.1109 /$ ICDCSW.2009.59

Wang, Z., H. Li, X. Shen, X. Sun and Z. Wang, 2008. Tracking and predicting moving targets in hierarchical sensor networks. Proceedings of the IEEE International Conference on Networking, Sensing and Control, Apr. 6-8, IEEE Xplore Press, Sanya, pp: 1169-1173. DOI: 10.1109/ICNSC.2008.4525393

Wang, Z., W. Lou, Z. Wang, J. Ma and H. Chen, 2013. A hybrid cluster-based target tracking protocol for wireless sensor networks. Int. J. Distrib. Sensor Netw. DOI: 10.1155/2013/494863

Yan, D. and J. Wang, 2010. Dynamic cluster formation Algorithm Target Tracking-oriented. Proceedings of the IEEE International Conference on Computer Design and Applications, Jun. 25-27, IEEE Xplore Press, Qinhuangdao, pp: 357-360. DOI: 10.1109/ICCDA.2010.5541079

Yang, H. and B. Sikdar, 2003. A protocol for tracking mobile targets using sensor networks. Proceedings of the First IEEE International Workshop on Sensor Network Protocols and Applications, May 11-11, IEEE Xplore Press, NY, USA, pp: 71-81.

DOI: $10.1109 /$ SNPA.2003.1203358
Yick, J., B. Mukherjee and D. Ghosal, 2008. Wireless sensor network survey. J. Comput. Netw., 52: 2292-2330. DOI: 10.1016/j.comnet.2008.04.002

Younis, M., I.F. Senturk, K. Akkaya, S. Lee and F. Senel, 2014. Topology management techniques for tolerating node failures in wireless sensor networks: A survey. Comput. Netw., 58: 254-283. DOI: 10.1016/j.comnet.2013.08.021

Younis, O. and S. Fahmy, 2004. Distributed clustering in ad-hoc sensor networks: A hybrid, energy-efficient approach. IEEE Trans. Mob. Comput., 3: 366-379.

Zhang, D., F. Xia, Z. Yang, L. Yao and W. Zhao, 2010. Localization technologies for indoor human tracking. Proceedings of the 5th International Conference on Future Information Technology (FutureTech), May 21-23, IEEE Xplore Press, Busan, pp: 1-6.

DOI: 10.1109/FUTURETECH.2010.5482731

Zhenga, J., M.Z.A. Bhuiyan, S. Liang, X. Xing and G. Wang, 2014. Auction-based adaptive sensor activation algorithm for target tracking in wireless sensor networks. Future Generat. Comput. Syst., 39: 88-99. DOI: 10.1016/j.future.2013.12.014

Zhu, C., L. Shu, T. Hara, L. Wang and S. Nishio et al., 2011. A survey on communication and data management issues in mobile sensor networks. Wireless Commun. Mob. Comput., 14: 19-36. DOI: $10.1002 / \mathrm{wcm} .1219$ 